\title{
Anomalous Diffusion in Cardiac Tissue as an Index of Myocardial Microstructure
}

\author{
Alfonso Bueno-Orovio*, Irvin Teh, Jürgen E. Schneider, Kevin Burrage, and Vicente Grau
}

\begin{abstract}
Diffusion in biological tissues is known to be hindered by the structural complexity of the underlying medium. In the heart, improved characterisation on how this complexity influences acquired diffusion weighted signals is key to advancing our interpretation of diffusion magnetic resonance imaging, as well as to propose novel biomarkers to further characterise myocardial microstructure. In this work, we propose stretched Mittag-Leffler signal decay models for the quantification of the anomalous decay observed in acquired diffusion weighted signals. Our results, analysed in ex vivo healthy, fixed rat ventricles, indicate that such a representation suffices to capture the anomalous signal decay observed in the myocardial syncytium. The subdiffusive order of signal decay is shown to encode independent information to that encapsulated by standard diffusion tensor metrics, and thus may provide additional information on tissue microstructure. Moreover, subdiffusion gradients are shown to be indicative of the total structural heterogeneity spanning the left ventricular wall, which includes progressive myolaminae branching and spatially varying densities of perimysial collagen, microvasculature and adipose tissue. The proposed approach may therefore have important implications for the characterisation of tissue microstructure, both in cardiac and other tissue types.
\end{abstract}

Index Terms-Anomalous diffusion, cardiac tissue, structural heterogeneity, ventricular microstructure.

\section{INTRODUCTION}

D IFFUSION magnetic resonance imaging (MRI) is a noninvasive imaging technique that is sensitive to the magnetisation of molecules undergoing diffusion. As the motion

Manuscript received February 08, 2016; revised March 23, 2016; accepted March 24, 2016. Date of publication May 02, 2016; date of current version August 30, 2016. This work is supported by the Engineering and Physical Sciences Research Council (EP/J013250/1), Biotechnology and Biological Sciences Research Council (BB/I012117/1), Wellcome Trust (100246/Z/12/Z) and the British Heart Foundation (BHF) Centre for Research Excellence (RE/13/1/ 30181) and BHF New Horizon Grant (NH/13/30238), U.K. J. E. Schneider is a BHF Senior Basic Science Research Fellow (FS/11/50/29038). The authors acknowledge a Wellcome Trust Core Award (090532/Z/09/Z). Asterisk indicates corresponding author.

*A. Bueno-Orovio is with the Department of Computer Science, University of Oxford, OX1 3QD Oxford, U.K., and also with the Oxford BHF Centre of Research Excellence (e-mail: alfonso.bueno@cs.ox.ac.uk).

I. Teh and J. E. Schneider are with the Division of Cardiovascular Medicine, University of Oxford, OX3 9DU Oxford, U.K., and the Oxford BHF Centres of Research Excellence and Regenerative Medicine (e-mail: irvin.teh@cardiov. ox.ac.uk, jurgen.schneider@(cardiov.ox.ac.uk).

$\mathrm{K}$. Burrage is with the Department of Computer Science, University of Oxford, OX1 3QD Oxford, U.K., and also with the School of Mathematical Sciences and ARC Centre of Excellence for Mathematical and Statistical Frontiers, Queensland University of Technology, Brisbane QLD 4001, Australia (e-mail: kevin.burrage@cs.ox.ac.uk).

V. Grau is with the Department of Engineering Science, University of Oxford, OX3 7QD Oxford, U.K., and also with the Oxford BHF Centre of Research Excellence (e-mail: vicente.grau@eng.ox.ac.uk).

Color versions of one or more of the figures in this paper are available online at http://ieeexplore.ieee.org.

Digital Object Identifier 10.1109/TMI.2016.2548503 of the diffusing molecules is hindered and restricted by tissue structures, this is a robust procedure for mapping structural features and in characterising tissue heterogeneity and anisotropy. In the particular case of cardiac tissue, diffusion tensor imaging (DTI) reconstruction of myofibre architecture has been validated ex vivo against histological studies [1]-[4]. In spite of its current limitations and associated difficulties in the in vivo heart, reproducibility has already been established in vivo [5], and novel applications of DTI as a clinical tool are becoming increasingly significant, such as for the diagnosis of regional impairment in transverse ventricular relaxation [6].

DTI analysis allows for the estimation of the initial and approximately monoexponential decay exhibited by the acquired signal at small diffusion weighting gradients. However, it is recognised that, under large diffusion weighting gradients, signal attenuation in biological tissues is less prominent than the predicted monoexponential [7]-[11]. This type of anomalous signal decay also holds for cardiac tissue [12], [13]. Consequently, the diffusion behaviour has been modelled with a biexponential form [7], [12]. While it may be tempting to theorise that the fast and slow components correspond to extracellular and intracellular compartments, this does not agree with the actual cell fractions [12], [14], and further investigations are still needed.

Recently, stretched exponential and Mittag-Leffler models have been proposed as alternatives for describing anomalous signal decay, as representations of superdiffusion and subdiffusion processes, respectively [15], [16]. Unlike the biexponential model, which to some extent assumes distinct standard diffusion properties in the two compartments, these models incorporate a broad and continuous distribution of diffusion properties, and may describe transport processes influenced by the multiple length scales of a heterogeneous medium at sub-voxel resolution. In addition, recent work in neural tissue has shown anomalous superdiffusion to be influenced by local magnetisation gradients, whilst subdiffusion reflects the multi-scale degree of disorder of local tissue microstructure [9]. This allows for a closer interpretation of model output in terms of tissue microstructure than those obtained by other models.

In this work, we investigate the response of diffusion weighted signals in healthy, fixed rat ventricles under a wide range of applied magnetic gradient amplitudes. The capabilities of generalised stretched Mittag-Leffler models are then explored to capture the anomalous decay observed in the signals. In addition, we present an intra-ventricular and inter-sample analysis of anomalous diffusion against standard DTI metrics, confirming the ability of the subdiffusion index to explain the total structural heterogeneity known to span the left ventricular 
wall. Our results therefore suggest the potential of the proposed index as a novel biomarker for further characterisation of cardiac tissue microstructure.

\section{METHODS}

\section{A. Models of Anomalous Diffusion Signal}

Assuming the Bloch-Torrey equation for the magnetisation of water protons, together with the Stejskal-Tanner sequence, the amplitude of the acquired signal follows the decay

$$
S / S_{0}=\exp \left(-b \cdot \mathbf{g}^{T} \mathbf{D g}\right)
$$

where $S_{0}$ is the baseline signal intensity, $\mathbf{D}$ is a positive definite symmetric diffusion tensor, and $b=(\gamma|\mathbf{G}| \delta)^{2}(\Delta-\delta / 3)$ is the $b$ value. Here the diffusion-weighting is applied with a pair of unipolar gradient waveforms of duration $\delta$, separation $\Delta$, amplitude $|\mathbf{G}|$, and unit direction $\mathbf{g}=\mathbf{G} /|\mathbf{G}|$.

As a generalisation of this monoexponential decay, stretched exponential models have been proposed based on fractal considerations of tissue microstruture [17]. Accounting for the anisotropic diffusion tensor, these models are formulated as

$$
S / S_{0}=\exp \left(-\left(b \cdot \mathbf{g}^{T} \mathbf{D g}\right)^{\alpha}\right), \quad 0<\alpha \leq 1,
$$

which recovers (1) for $\alpha=1$. Values of $\alpha<1$ yield a power law signal decay, as characteristic of superdiffusion [11], [18].

An alternative approach to characterising anomalous signal decay is by means of the generalised exponentials given by

$$
S / S_{0}=E_{\beta}\left(-b \cdot \mathbf{g}^{T} \mathbf{D g}\right), \quad 0<\beta \leq 1,
$$

where $E_{\beta}(z)=\sum_{k=0}^{\infty} z^{k} / \Gamma(1+\beta k)$ and $\Gamma(\cdot)$ are the Mittag-Leffler and Gamma functions. Mittag-Leffler functions arise naturally in the solution of diffusion equations where the time operator is replaced by a fractional counterpart to account for increased lag times, where physical connections with microstructure have been established for example in the context of porous media [19]. For $\beta=1, \Gamma(1+k)=k$ !, and $E_{1}(z)$ is the exponential. Values of $\beta<1$ yield a heavy tail decay, as characteristic of memory effects in subdiffusion [20], [21].

Finally, both models can be combined to give a signal decay based on coexisting superdiffusive and subdiffusive processes, in the form of a stretched Mittag-Leffler model

$$
S / S_{0}=E_{\beta}\left(-\left(b \cdot \mathbf{g}^{T} \mathbf{D g}\right)^{\alpha}\right), \quad 0<\alpha, \beta \leq 1 .
$$

Here, we additionally compare the anomalous signal decay model (4) against the biexponential tensor model [12]

$$
S / S_{0}=\omega_{f} \exp \left(-b \cdot \mathbf{g}^{T} \mathbf{D}^{\mathbf{f}} \mathbf{g}\right)+\omega_{s} \exp \left(-b \cdot \mathbf{g}^{T} \mathbf{D}^{\mathbf{s}} \mathbf{g}\right),
$$

where $\mathbf{D}^{\mathbf{f}}$ and $\mathbf{D}^{\mathbf{s}}$ are the diffusion tensors of the fast and slow components, with weights $\omega_{f}$ and $\omega_{s}$, respectively.

\section{B. Data Acquisition}

Hearts were excised from four female Sprague-Dawley rats (hereafter denoted as ventricles V1 to V4). The hearts were swiftly cannulated via the aorta for coronary perfusion in Langendorff mode at a constant pressure of $80 \mathrm{~mm} \mathrm{Hg}$ and $37^{\circ} \mathrm{C}$.
After the initial wash using modified Krebs-Henseleit buffer (in $[\mathrm{mM}]: \mathrm{NaCl} 118.5, \mathrm{NaHCO}_{3} 25.0, \mathrm{KCl} 4.75, \mathrm{KH}_{2} \mathrm{PO}_{4}$ 1.18, $\mathrm{MgSO}_{4} 1.19$, D-glucose 11.0, and $\mathrm{CaCl}_{2} 1.41$ ), the heart was cardioplegically arrested using a high- $\mathrm{K}^{+}$solution (in $[\mathrm{mM}]$ : $\mathrm{NaCl}$ 125.0; $\mathrm{KCl} 20.0 ; \mathrm{MgCl}_{2}$ 1.0; HEPES 5.0; D-glucose 11.0; $\mathrm{CaCl}_{2}$ 1.8). All solutions were titrated to $\mathrm{pH} 7.4$ and gassed with $95 \% \mathrm{O}_{2} / 5 \% \mathrm{CO}_{2}$. The hearts were perfused with low osmolality Karnovsky's fixative (Solmedia, Shrewsbury, UK), then stored in the same fixative at $4^{\circ} \mathrm{C}$. Samples were rinsed with cacodylate buffer and embedded in 1\% agarose gel for MRI. All animal work was conducted in accordance with the UK Home Office Guidance on the Operation of Animals (Scientific Procedures) Act of 1986, and approved by the University of Oxford ethical review board.

MRI was performed on a $9.4 \mathrm{~T}$ preclinical MRI scanner (Agilent, CA, USA) with a shielded gradient system (maximum gradient strength, $1 \mathrm{~T} / \mathrm{m}$; rise time, $130 \mu \mathrm{s}$ ), and transmit/receive birdcage coil (inner diameter, $20 \mathrm{~mm}$; Rapid Biomedical, Rimpar, Germany). Diffusion spectrum imaging (DSI) data were acquired with $3 \mathrm{D}$ spin echo echo planar imaging (SEEPI) in four segments with no signal averaging, with: TR/TE, $1000 / 18 \mathrm{~ms}$; echo train length, 20 ; FOV, $24 \times 20 \times 20 \mathrm{~mm}$; resolution, $250^{3} \mu \mathrm{m}^{3}$; acquisition time, $47 \mathrm{~h}$. Forward and reversed readout polarity data were acquired to correct for inconsistencies between odd and even lines of $k$-space [22]. Unipolar diffusion sensitising gradients were used: $\delta, 5 \mathrm{~ms} ; \Delta, 12 \mathrm{~ms} ; b_{\max }$, $10000 \mathrm{~s} / \mathrm{mm}^{2} . Q$-space was sampled in $257 \mathrm{steps}$ in a $3 \mathrm{D}$ grid circumscribed by half a sphere, taking advantage of symmetry in $q$-space to halve the acquisition time. DSI data were also acquired in a $20 \mathrm{~mm}$ tube filled with cyclooctane (Sigma-Aldrich, MO, USA) with identical diffusion encoding parameters. Here, TR/TE, 1000/24 ms; echo train length, 32; FOV, $24^{3} \mathrm{~mm}^{3}$; resolution, $375^{3} \mu \mathrm{m}^{3}$; acquisition time, $19 \mathrm{~h}$.

\section{Signal Decay Fitting}

The estimation of the diffusive parameters in the anomalous signal decay model was performed on a voxel-by-voxel basis in a two-steps algorithm. Firstly, the average diffusion tensor D was calculated by means of the H-matrix approach, using the subset of acquired data up to $b=1000 \mathrm{~s} / \mathrm{mm}^{2}$ in order to approximate the monoexponential decay at low $b$ values. Secondly, the complete range of $b$ values was used to fit the fractional order parameters in (4), using a steepest-descent trust-region algorithm for constrained optimisation, with prescribed tolerance of $10^{-6}$. Parameter bounds were taken as $0<\alpha, \beta \leq 1$, with initial values $\alpha=\beta=1$, representative of standard diffusion. Similarly, the biexponential tensor model was adjusted by initialising $\mathbf{D}^{\mathbf{f}}$ and $\mathbf{D}^{\mathbf{s}}$ to the average diffusion tensor $\mathbf{D}$, with $\omega_{f}=\omega_{s}=0.5$. An unconstrained optimisation was then performed on the elements of $\mathbf{D}^{\mathbf{f}}$ and $\mathbf{D}^{\mathbf{s}}$, whilst ensuring $0 \leq w_{f} \leq 1, \omega_{s}=1-\omega_{f}$. Fitted results were insensitive to the choice of initial values.

All calculations were implemented in MATLAB R2012b (MathWorks Inc, Natick, MA). Evaluation of the Mittag-Leffler function was performed following [23], as available in MATLAB CEnTRAL (File ID \#48154). The routines were vectorised and particularised for negative real arguments $(\sim 200$ code speed-up). Analysis of a full dataset on a standard desktop 
took $\sim 20$ and $\sim 100 \mathrm{~min}$ for the anomalous signal decay and biexponential tensor models, respectively.

\section{Data Analysis}

Signal decays are presented as functions in the $q$-space $b \cdot \mathbf{g}^{T} \mathbf{D g}$, which combines all acquisition points into a single attenuation curve regardless of the diffusion weighting direction. This choice of abscissa also enables a more straightforward inter-sample comparison, as reflected by a same monoexponential decay regardless of local tissue diffusivities.

Diffusion tensor eigenvalues were calculated on a voxel-byvoxel basis. From these, the mean apparent diffusion coefficient $(\overline{\mathrm{ADC}})$ and fractional anisotropy (FA) were computed, in order to analyse possible correlations with the estimated anomalous model parameters. Estimated parameters lying outside their 1.5 $\times$ inter-quartile bounds were identified as outliers, and subsequently removed to minimise possible influences on the results. Outliers were typically localised on the segmented boundaries due to partial volumes, and represented $<1 \%$ of the total of analysed voxels.

Additionally, the left ventricular endocardial and epicardial surfaces were approximated by prolate spheroids, and the resulting volumes subdivided following the standardised segmentation of the American Heart Association (AHA) [24]. Supporting research data are available upon request.

\section{RESULTS}

\section{A. Acquired Diffusion Signals Exhibit a Marked Anomalous Decay in Cardiac Tissue}

Acquired diffusion signals in cardiac tissue exhibited a marked anomalous attenuation at large $b$ values, in contrast to the monoexponential decline predicted by standard diffusion theory. This is shown in Fig. 1, for indicated voxels within the mid-ventricular slice of the preparations. The anomalous signal decay was independent of ventricular location and baseline signal intensity, and consistent across all data sets. This is in contrast to the response displayed by an isotropic calibration phantom (Fig. 1, bottom), which exhibited a standard diffusion attenuation within the whole range of analysed $b$ values.

Our choice of abscissas also underlies the smaller number of aggregated points in the isotropic phantom compared to the rest of panels, due to its smaller diffusivity compared to ventricular tissue (see Table I). This yields a smaller $b \cdot \mathbf{g}^{T} \mathbf{D g}$ in spite of being scanned in the same range of $b$ values.

\section{B. Subdiffusion Dominates Anomalous Decay in Cardiac Tissue}

Results on the approximation of the observed signal decays by the anomalous signal decay model (4) are presented in Fig. 2, as shown for the indicated septal voxel of ventricle V1 in Fig. 1 as a representative signal. The role of anomalous order parameters on model output is illustrated in Fig. 2(a)-(b). Both the superdiffusive $(\alpha)$ and subdiffusive $(\beta)$ components induce an increasing degree of anomalous decay for decreasing values of their respective orders. However, marked differences in signal decay are observed between both anomalous parameters, with much steeper signal decays at high $b$ values in the
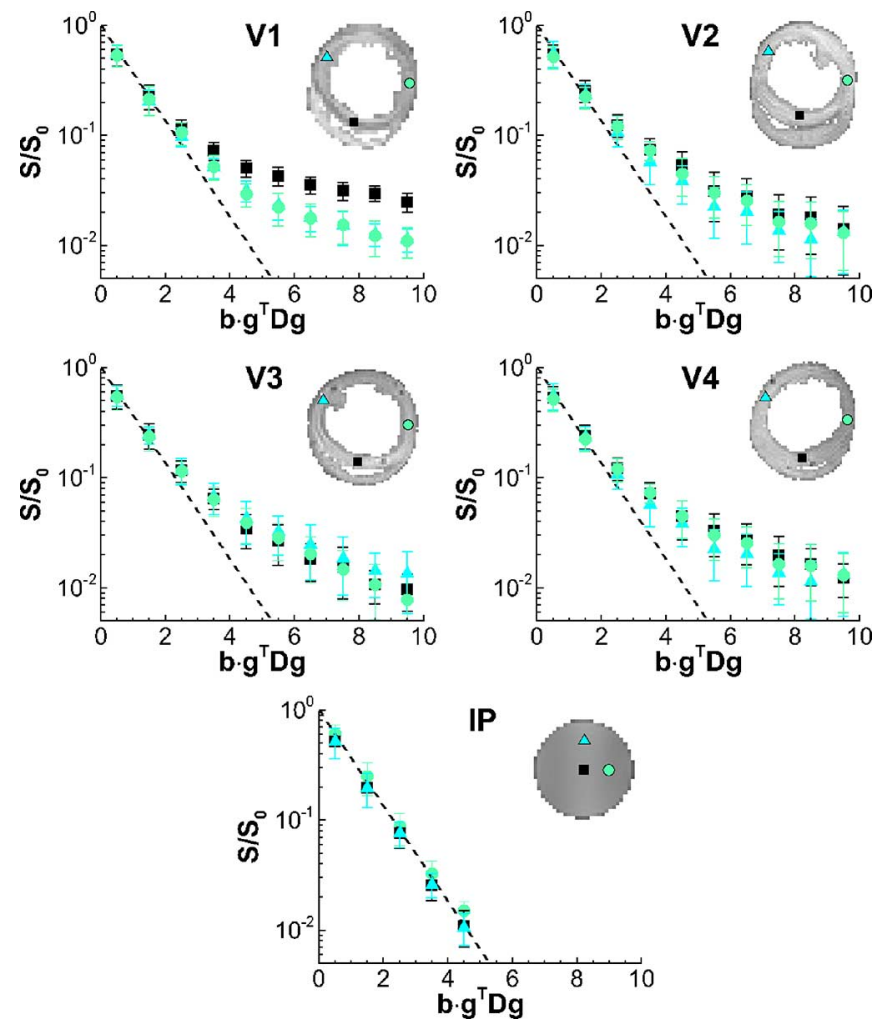

Fig. 1. Experimental evidence of anomalous diffusion in cardiac tissue V1-V4: rat ventricles; IP: isotropic phantom. Normalised baseline intensities $S_{0}$ in the central slice of the data sets and signal decays for the indicated voxels are shown. In cardiac tissue, signal decay shows marked deviations from the monoexponential decay predicted by standard diffusion theory (dashed lines). Experimental data (filled symbols) are presented as mean $\pm \mathrm{SD}$.

TABLE I

Mean \pm SD SUMMARY OF AGGREGATED DIFFUSION PARAMETERS. V1-V4: RAT VENTRICleS; IP: ISOTROPIC PHANTOM. ADC IN $\mathrm{mm}^{2} / \mathrm{s} ; \mathrm{FA}, \alpha$ AND $\beta$, DIMENSIONLESS

\begin{tabular}{cccccc} 
& $\overline{\text { ADC }}\left(\times 10^{-3}\right)$ & FA & $\alpha$ & $\beta$ \\
\hline V1 & $1.025 \pm 0.161$ & $0.197 \pm 0.061$ & $0.998 \pm 0.001$ & $0.910 \pm 0.031$ \\
V2 & $1.129 \pm 0.241$ & $0.272 \pm 0.098$ & $0.976 \pm 0.025$ & $0.884 \pm 0.017$ \\
V3 & $0.935 \pm 0.178$ & $0.318 \pm 0.094$ & $0.967 \pm 0.030$ & $0.898 \pm 0.020$ \\
V4 & $0.834 \pm 0.147$ & $0.372 \pm 0.088$ & $0.983 \pm 0.019$ & $0.895 \pm 0.024$ \\
IP & $0.522 \pm 0.022$ & $0.028 \pm 0.026$ & $0.981 \pm 0.018$ & $0.995 \pm 0.004$
\end{tabular}

stretched exponential $(\alpha)$ than in the Mittag-Leffler $(\beta)$ model compared to the experimental data. Model behaviour at low $b$ values is further highlighted in the panel insets. In particular, the stretched exponential model significantly overestimates the initial signal decline for decreasing values of $\alpha$ (Fig. 2(a)) . On the other hand, such a signal overshoot at low $b$ values is not present in the generalised Mittag-Leffler model (Fig. 2(b)).

Residuals in the $\ell_{2}$-norm in the approximation of the considered data are shown in Fig. 2(c) for different combinations of parameters $\alpha$ and $\beta$. Starting from standard diffusion $(\alpha=\beta=1$, left corner of the plot), residuals show a marked initial reduction in the approximation error for decreasing values of both parameters, then quickly raising as the anomalous decay becomes excessively pronounced. For the signal decay under consideration, the residuals map shows a unique global minimum along the axis representing the subdiffusive regime $(0<\beta<1$, $\alpha=1$ ). The convergence history of the proposed optimisation 

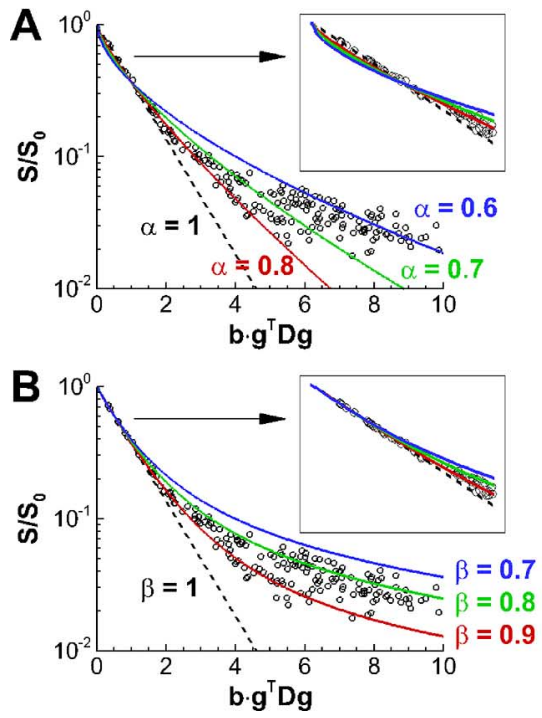

C

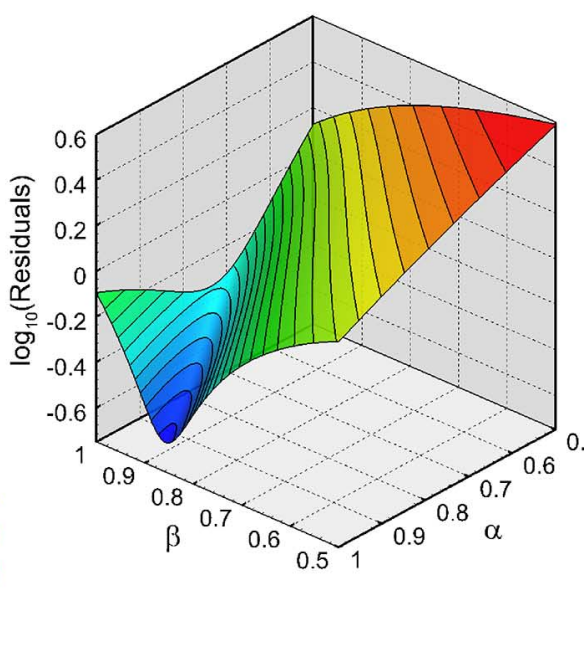

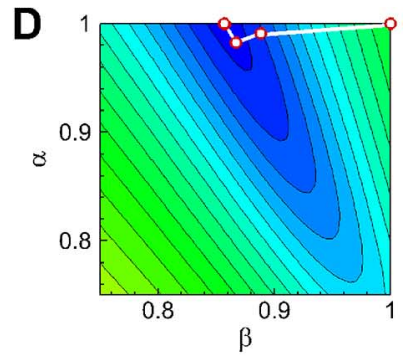

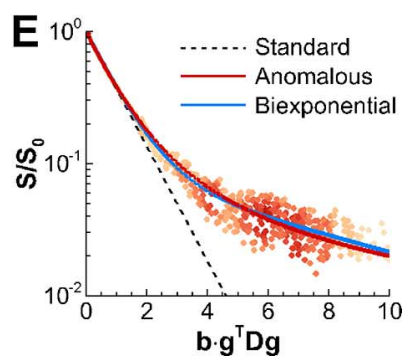

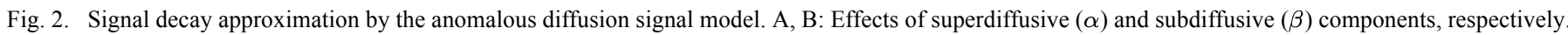

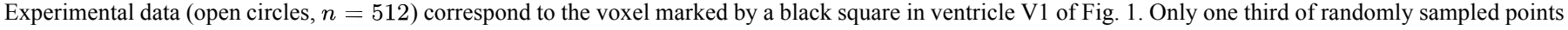

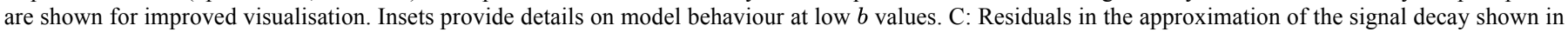

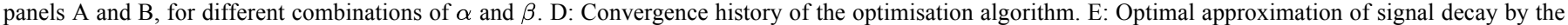
anomalous diffusion model $(\beta=0.857, \alpha=1)$, compared against a biexponential fit of the data. Points are colour-coded by density distribution.

algorithm is illustrated in Fig. 2(d), attaining the global minimum in four optimisation steps. The optimal approximation of this signal decay by the anomalous diffusion model $(\beta=0.857$, $\alpha=1$ ) is presented in Fig. 2(e).

Fig. 3 shows spatially resolved maps of diffusion parameters for the anomalous signal decay model given by (4). These include the mean apparent diffusion coefficient ( $\overline{\mathrm{ADC}}$ ) as a metric for the diffusion tensor, as well as the respective subdiffusive $(\beta)$ and superdiffusive $(\alpha)$ orders. A manifest subdiffusion $(\beta<1)$ is observed throughout all ventricles, in the absence of a superdiffusive component ( $\alpha \sim 1)$. Our findings hence suggest that subdiffusion dominates and suffices to explain the anomalous signal decay as observed in cardiac tissue. In fact, superdiffusive exponents $\alpha$ in the order of those reported in Table I would only yield minor deviations from a monoexponential decay. Taken together, these results support reducing the full anomalous diffusion model to its subdiffusive counterpart (3). For these reasons, we will focus our analysis in subsequent sections on the subdiffusive order $(\beta)$.

\section{Anomalous Diffusion Encodes Independent Information From Standard Diffusion Metrics}

To exclude for anomalous diffusion being underpinned by local tissue diffusivities, we performed a quantitative analysis of diffusion metrics interdependencies, as presented in Fig. 4. In terms of standard DTI metrics, a marked negative correlation was found in our data between the apparent diffusion coefficient and fractional anisotropy (Fig. 4(a)), highlighting as expected bigger diffusion displacements (larger $\overline{\mathrm{ADC}}$ ) at more isotropic positions of the tissue (smaller FA).

On the other hand, only a modest trend towards more anomalous behaviours (smaller $\beta$ ) was observed for increasing diffusivities (Fig. 4(b)). A similar trend was noticed when examining the dependence of the subdiffusive anomalous order with respect to the FA metric (Fig. 4(c)). However, the weak coeffi-

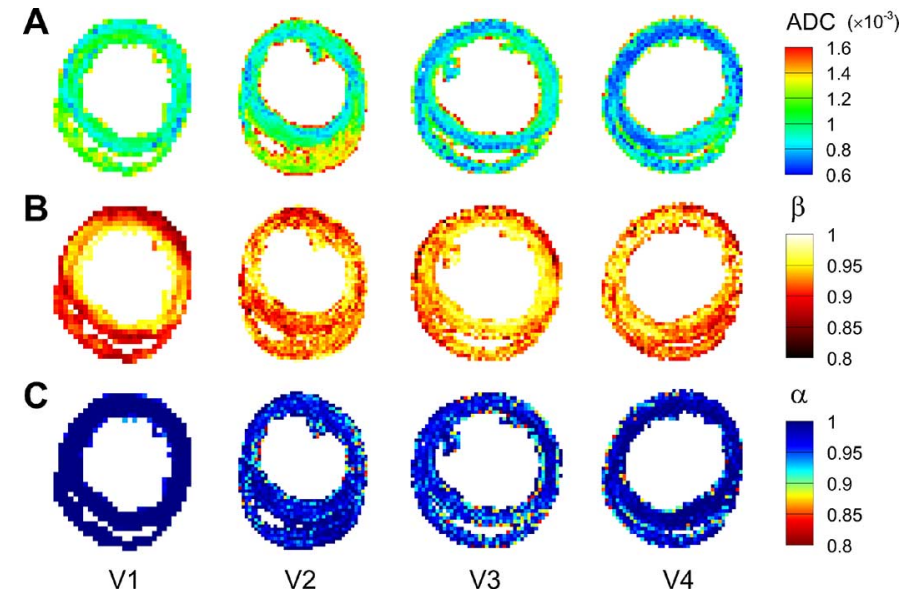

Fig. 3. Spatially resolved maps of diffusion parameters in the mid-ventricular slices of the different data sets. A: Mean apparent diffusion coefficient ( $\overline{\mathrm{ADC}}$ ). B: Subdiffusive order $(\beta)$. C: Superdiffusive exponent $(\alpha)$. A clear subdiffusion component $(\beta<1)$ is observed throughout the entire ventricles, in the absence of superdiffusion $(\alpha \sim 1)$. $\overline{\mathrm{ADC}}$ in $\mathrm{mm}^{2} / \mathrm{s} ; \alpha$ and $\beta$, dimensionless.

cients of determination $\left(r^{2}\right)$ attained in both cases indicate that only a minimum fraction of the variance observed in $\beta$ can be explained in terms of these metrics.

Equivalent results were obtained by using higher order regression models and/or additional DTI metrics (such as rational anisotropy, independent eigenvalues magnitudes, eigenvalues ratios, or combinations of the above), and in the different ventricles. Altogether, this supports that the subdiffusive order $\beta$ encodes independent information on tissue microstructure to that encapsulated by the diffusion tensor itself.

\section{Anomalous Diffusion Captures the Structural Heterogeneity of the Left Ventricular Wall}

Apico-basal diffusion gradients were further studied in the ventricular datasets (Fig. 5(a)). The analysis of standard DTI 

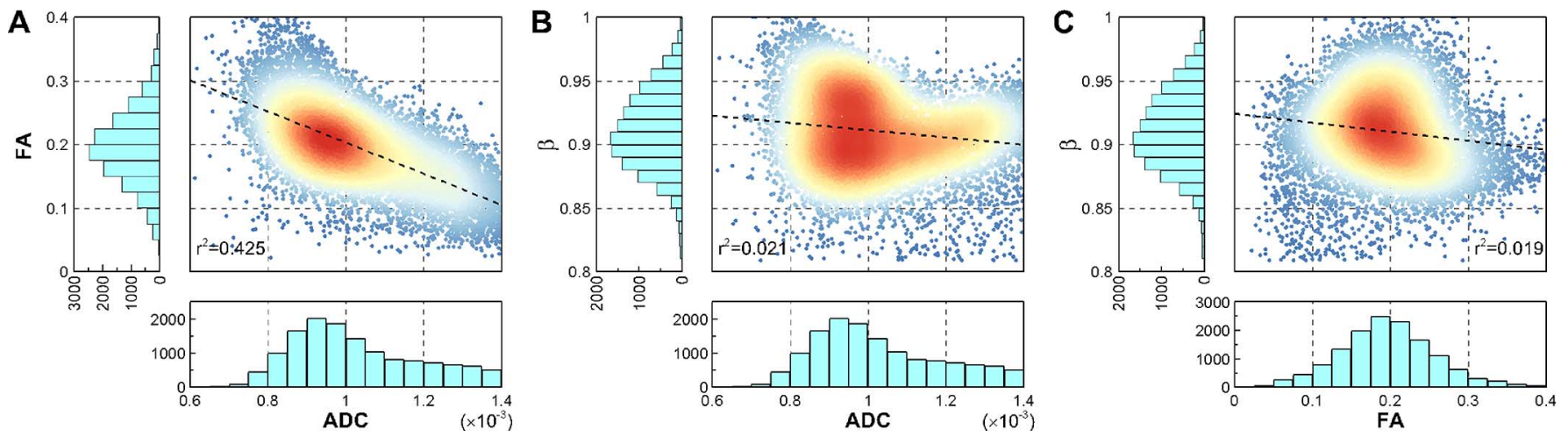

Fig. 4. Correlation analysis between diffusion metrics. A: Fractional anisotropy (FA) vs. mean apparent diffusion coefficient $(\overline{\mathrm{ADC}})$. B: Subdiffusive anomalous $\operatorname{order}(\beta)$ vs. ADC. C: $\beta$ vs. FA. Results and associated histograms are aggregated for the whole ventricle V1, with points colour-coded by their two-dimensional density distributions. Dashed lines represent linear regression fits between the studied variables. $\overline{\mathrm{ADC}}$ in $\mathrm{mm}^{2} / \mathrm{s} ;$ FA and $\beta$, dimensionless.

A

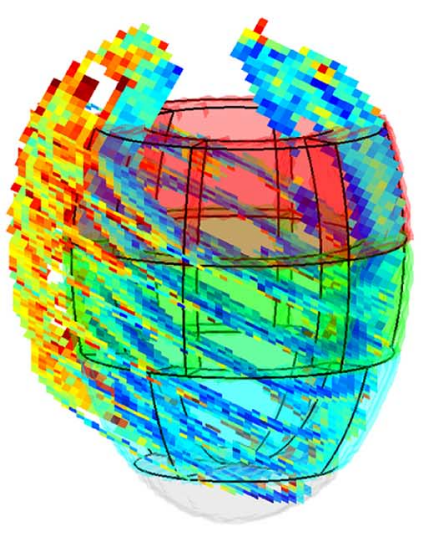

B

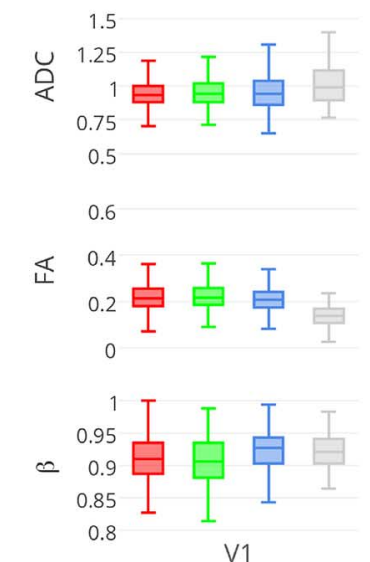

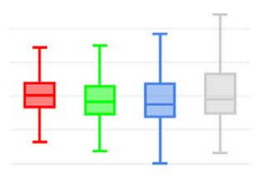
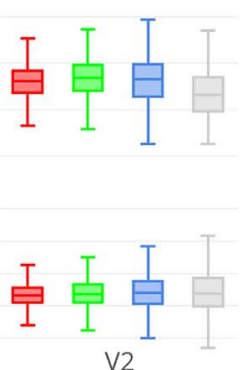
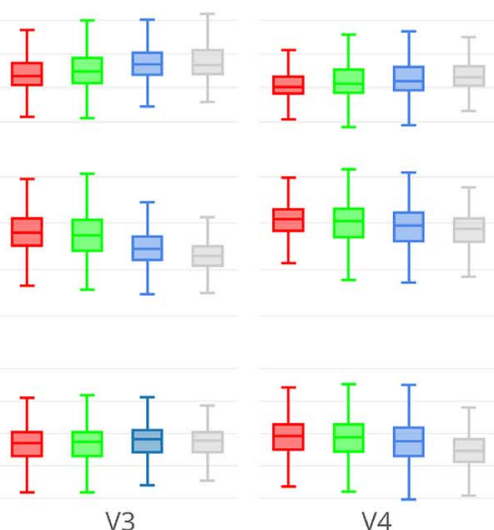

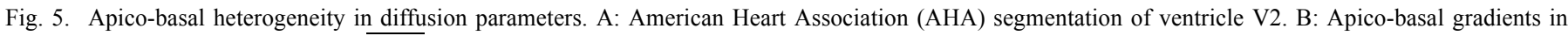

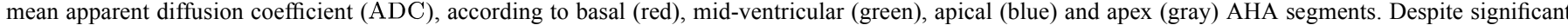

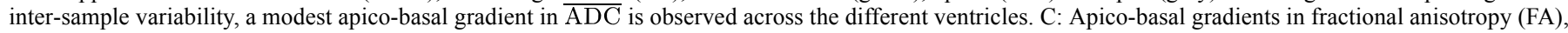

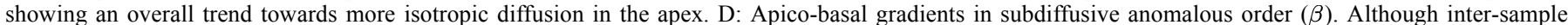
differences are also observed, apico-basal distributions are more uniform than in $\overline{\mathrm{ADC}}$ and FA. $\overline{\mathrm{ADC}}$ in $\mathrm{mm}{ }^{2} / \mathrm{s} ; \mathrm{FA}$ and $\beta$, dimensionless.

metrics (Fig. 5(b)-(c)) reveals a significant level of inter-sample variability, as previously summarized in Table I. Despite these individual differences, overall trends towards slightly larger mean diffusivities (larger $\overline{\mathrm{ADC}}$, Fig. 5(b)) and a more isotropic diffusion (smaller FA, Fig. 5(c)) were identified in the apex compared to the basal segments across all ventricles, with the exception of ventricle V2, in which the positive apico-basal gradient in $\overline{\mathrm{ADC}}$ was not observed. An equivalent analysis is presented in Fig. 5(d) for the subdiffusive order $(\beta)$. Despite significant differences being present as well, both inter-sample and intra-ventricular variability in $\beta$ were less pronounced than in standard DTI metrics. Apico-basal distributions of $\beta$ were also more uniform than in $\overline{\mathrm{ADC}}$ and FA, without consistent apico-basal patterns being identified across the ventricles.

Transmural heterogeneities in diffusion parameters were also investigated (Fig. 6(a)). Aggregated results for the considered metrics are presented in Fig. 6(b)-(d) with respect to the normalised distance to the endocardial cavity, e. In general, a convex response in mean diffusivities is exhibited across the ventricular wall, with $\overline{\mathrm{ADC}}$ first decreasing in the central region and then approaching similar values in the epicardium than those in the endocardium (Fig. 6(b)). A mirrored trend to the behaviour of $\overline{\mathrm{ADC}}$ is observed in FA (Fig. 6(c)), due to the negative correlation between both metrics, as discussed previously (Fig. 4(a)). Diffusion was therefore found to be moderately more anisotropic (larger FA) at central transmural locations, decreasing towards more isotropic diffusion in the subepicardial ventricular layer. These results are hence in agreement with the reported progressive branching in ventricular laminar structure as a function of wall depth [25]-[27], and the absence of myolaminae from much of the subepicardium [26], [28], respectively. The only exception to these transmural trends was noted again in ventricle V2, which falls however within the limits of inter-individual structural variability [29].

In terms of $\beta$, a monotonic trend towards more anomalous diffusion was identified with respect to wall depth (Fig. 6(d)). Hence, the subdiffusive order is also able to capture the increasingly branching structure of the ventricular wall. However, and contrary to the FA metric, a marked inflection in the values of $\beta$ is not observed in the subepicardial layer. Although the laminar arrangement of the ventricular wall is lost in most of this region, dense and sparsely interconnected arrays of perimysial collagen have been reported in the subepicardium [28], which is also known to be richer in microvasculature and adipose tissue 
A

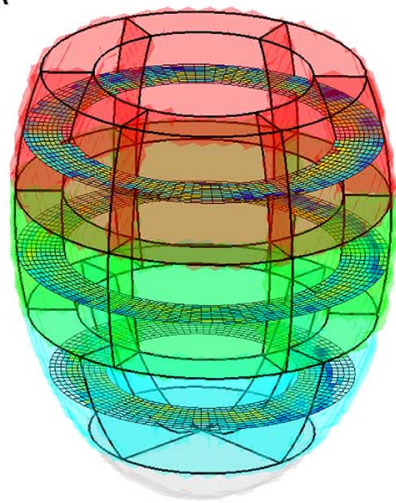

B

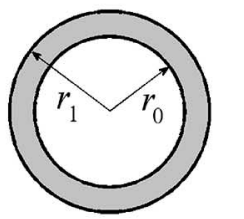

$e=\frac{r-r_{0}}{r_{1}-r_{0}}$

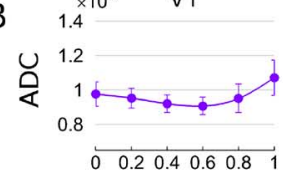

C $\quad 0.5$

$\ll \quad 0.4$

世 0.3

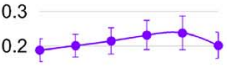

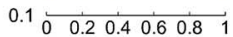

D

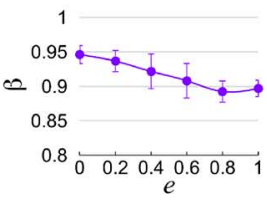

V2

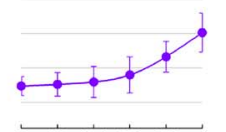

\begin{tabular}{lllllll}
\hline & 1 & 1 & & 1 & & \\
0 & 0.2 & 0.4 & 0.6 & 0.8 & 1
\end{tabular}

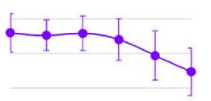

\begin{tabular}{llllll}
\hline 0 & 0.2 & 0.4 & 0.6 & 0.8 & 1
\end{tabular}

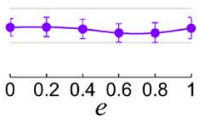

V3

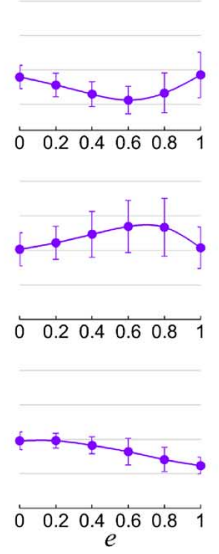

V4
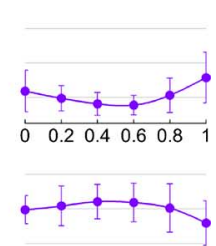

$\begin{array}{llllll}0 & 0.2 & 0.4 & 0.6 & 0.8\end{array}$

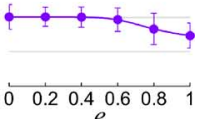

Fig. 6. Transmural heterogeneity in diffusion parameters. A: Central slices in the basal (red), mid-ventricular (green) and apical (blue) AHA segments are examined, as shown for ventricle V3. Results are presented as mean $\pm \mathrm{SD}$ with respect to the normalised endocardial distance, $e$. B: In general, a convex response in mean diffusivities is observed, with smaller ADCs within the ventricular wall and similar subepicardial $(e=1)$ and subendocardial $(e=0)$ values. C: Fractional anisotropy (FA) exhibited a mirrored trend to that of $\overline{\mathrm{ADC}}$, also with similar subendocardial and subepicardial FA values. D: Overall, diffusion in the subepicardial layer exhibits a more anomalous behaviour $\left(\operatorname{smaller} \beta\right.$ ) than in the subendocardium. $\overline{\mathrm{ADC}}$ in $\mathrm{mm}^{2} / \mathrm{s}$; FA and $\beta$, dimensionless.
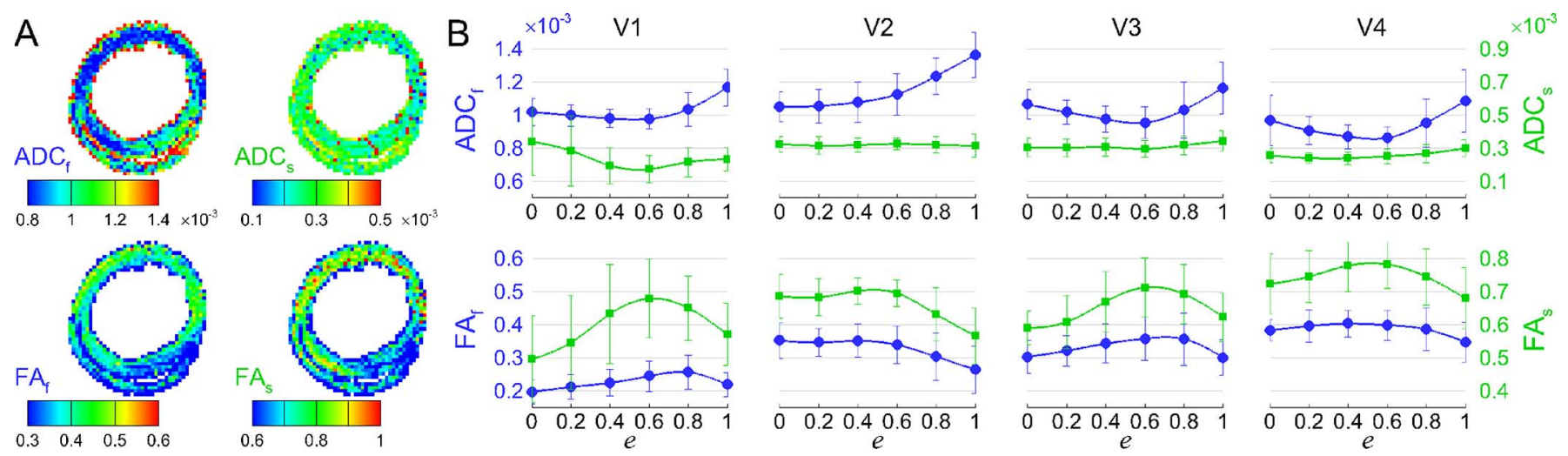

Fig. 7. Diffusion metrics in biexponential tensor models. A: Spatial maps of fast/slow mean diffusivity and fractional anisotropy $\left(\overline{\mathrm{ADC}}_{f} / \overline{\mathrm{ADC}}_{s}\right.$ and $\mathrm{FA} f f / \mathrm{FA}_{s}$, respectively) in one of the analysed ventricles (V4). B: Transmural distributions of the different diffusion metrics, showing similar trends to those of the monoexponential model in Fig. 6 . Results are presented as mean $\pm \mathrm{SD}$ with respect to the normalised endocardial distance, $e . \overline{\mathrm{ADC}}$ in $\mathrm{mm}^{2} / \mathrm{s} ; \mathrm{FA}$ dimensionless.

than the subendocardial layers [30]-[32]. Therefore, the subdiffusive order $\beta$ was found as the only metric that may be directly related to the total structural heterogeneity known to exist in the left ventricular wall.

\section{E. Comparison Against Biexponential Tensor Models}

We finally compare our results against biexponential tensor models, previously used to study anomalous signal decay in myocardial tissue [12]. Fig. 7 shows spatial maps and transmural trends of $\overline{\mathrm{ADC}}$ and $\mathrm{FA}$ for the estimated fast and slow diffusion tensors $\left(\overline{\mathrm{ADC}}_{f} / \overline{\mathrm{ADC}}_{s}\right.$ and $\mathrm{FA}_{f} / \mathrm{FA}_{s}$, respectively). The two-compartment model exhibits similar fast-diffusivities $\left(\overline{\mathrm{ADC}}_{f}\right.$ and $\left.\mathrm{FA}_{f}\right)$ than those obtained for the average diffusion tensor D (Fig. 6), whilst the smaller slow-component diffusivities account for the slower signal decay at large $b$ values. However, the metrics associated to the slow diffusion tensor provided no additional information in terms of structural heterogeneity, with $\overline{\mathrm{ADC}}_{s}$ exhibiting overall flat transmural distributions, and $\mathrm{FA}_{s}$ following similar trends to those of the fast component.

\section{Discussion}

In this study, we have evaluated generalised stretch-exponential and Mittag-Leffler models to capture and explain the anomalous signal decay observed in diffusion weighted signals in cardiac tissue in a wide range of $b$ values. The subdiffusive order $\beta$ has been shown to encode independent information to that encapsulated by standard DTI metrics, and thus may provide additional information in the characterisation of tissue microstructure. Whereas no marked apico-basal differences were found in the new anomalous diffusion index, transmural gradients in $\beta$ are indicative of the total structural heterogeneity known to span the left ventricular wall.

Our work constitutes the first application of stretched exponential and Mittag-Leffler models for the characterisation of anomalous diffusion in the heart. Other MRI studies to date have explored cardiac microstructure in ranges of $b$ values typically spanning from 1000 up to $2500 \mathrm{~s} / \mathrm{mm}^{2}$ [27], [33], [34]. Whilst this suffices to capture myocardial anisotropy, they fall within the initial monoexponential response of water diffusion. Our estimation of diffusion tensors in the $0-1000 \mathrm{~s} / \mathrm{mm}^{2}$ range indeed allows for a direct comparison with previous results on 
DTI eigenvalues and associated metrics [27], [33], [34], while minimising the observed difference between the estimated myolaminar architecture and histological studies [26].

Whilst the proposed model of anomalous signal decay is clearly phenomenological, other modelling approaches have contributed to the understanding of different microstructural aspects on signal attenuation from a biophysical perspective (e.g., myofibre diameters, intracellular volumes, membrane permeability or bundle packing densities) [35], [36]. However, unambiguous parameter estimation in these models is a difficult task, limiting they practical applicability. Alternative representations of signal decay, improving the agreement between experimental data and the fitting curves, might therefore be of practical interest as long as they are capable of exhibiting sensitivity to tissue features or microstructural changes.

In these terms, the full anomalous diffusion model considered here provides two additional parameters to further characterise tissue microstructure: the subdiffusive $(\beta)$ and superdiffusive $(\alpha)$ anomalous orders. Previous work in neural tissue has shown anomalous superdiffusion to be influenced by local magnetisation gradients, thus highlighting the interface between compartments with different magnetic susceptibility [9]. Unlike the brain, which is formed by highly localised regions of white and gray matter with dissimilar magnetisation properties (hence reflected by spatial varying maps in $\alpha$ ) [10], [37], the myocardial syncytium mostly consists of ventricular myocytes. This may underlie the almost uniform spatial distributions in $\alpha$ that we observe in our experimental datasets.

On the other hand, anomalous subdiffusion has been previously shown to reflect the multi-scale disorder of local tissue microstructure, both in tortuous materials as in heterogeneous tissues [9], [10], [37]. Theoretical and simulation studies have also shown anomalous subdiffusion to arise as a consequence of hindered transport in densely packed and heterogeneous structures, via particle collisions with the obstacle surfaces (such as microvasculature and cleavage planes in cardiac tissue), which act to increase diffusion lag times [38], [39]. Our findings on transmural gradients in $\beta$ are hence in agreement with the structural heterogeneity observed across the left ventricular wall, including progressive myolaminae branching and spatially varying densities of perimysial collagen, microvasculature and adipose tissue [25]-[32]. They therefore suggest that $\beta$ may provide additional information on tissue structure to that provided by conventional DTI metrics, as well as its potential as a novel biomarker for the characterisation of cardiac microstructure. In fact, recent MRI studies have reported a higher sensitivity of anomalous diffusion parameters to ischemic changes in brain than standard metrics [40]. Strong correlations with the assessment of liver fibrosis have also been established [41]. The extension of our approach may therefore have important implications for the characterisation of different disease states, both in cardiac or other tissue types.

Our choice of descriptions of anomalous signal decay is based on their direct link with superdiffusive and subdiffusive processes. As also contemplated here, two-compartment models have been also suggested, leading to a biexponential signal decay [7], [12]. A more sophisticated two-compartment framework is given in [42], where the signal decay is described by a system of coupled Bloch-Torrey equations. Unfortunately there is no closed form solution, and is difficult to accommodate anisotropy in the approach. Other anomalous diffusion frameworks include adding a kurtosis term (essentially a cubic term into the exponential component), not analysed here due to its known associated deviation of signal decay at large $b$ values [43]. Different stretched exponential models have also been proposed in the anisotropic case, for instance by fitting the data to three stretched exponentials aligned along the principal eigenvector axes [8]. Alternatively, our signal decay model contemplates anisotropy by means of a diffusion tensor with the same meaning as in the traditional setting.

Finally, tissue microstructure also affects cardiac electrical propagation. The results presented here are thus in agreement with our previous work [44], [45], where anomalous diffusion models were shown to recover key properties of cardiac conduction. However, in terms of potential theory, the secondary sources related to tissue inhomogeneities are associated with superdiffusion effects on the electric field. On the other hand, the physical principle underlying anomalous water diffusion in cardiac tissue is different, based on increased lag times due to the collisions in a tortuous environment [38], [39], hence resulting in a subdiffusion. This distinction in the underlying physical processes underpins the difference in their associated anomalous diffusion nature, in spite of both phenomena taking place in the same anatomical substrate. The development of a framework to connect both physical processes, informing simulations of the electrical behaviour of the heart from MRI estimates of tissue microstructure beyond myofibre orientation, remains as an open and exciting challenge for future investigations.

\section{A. Limitations}

In our acquisition sequence, the diffusion gradient duration was finite and not insignificant relative to the diffusion time. This violation of the short gradient pulse condition [46] may limit the sensitivity of the measurement to shorter length scales in tissue, although this may not be a problem in practice as shown in DSI [47]. This constitutes an interesting open question for future studies.

Our model encapsulates the multi-scale complexity of tissue microstructure into a single parameter, the subdiffusive order $\beta$. This makes it challenging to separate and to establish a physical connection with different microstructural contributors to anomalous signal decay. Whereas the proposed representation has been proven to capture and explain the anomalous signal decay observed in our data, the use of more sophisticated models [11], [48] may be explored in a future.

\section{REFERENCES}

[1] E. W. Hsu, A. L. Muzikant, S. A. Matulevicius, R. C. Penland, and C. S. Henriquez, "Magnetic resonance myocardial fiber-orientation mapping with direct histological correlation," Am. J. Physiol., vol. 274, pp. H1627-H1634, 1998.

[2] D. F. Scollan, A. Holmes, R. Winslow, and J. Forder, "Histological validation of myocardial microstructure obtained from diffusion tensor magnetic resonance imaging," Am. J. Physiol., vol. 275, pp. H2308-H2318, 1998

[3] A. A. Holmes, D. F. Scollan, and R. L. Winslow, "Direct histological validation of diffusion tensor MRI in formaldehyde-fixed myocardium," Magn. Reson. Med., vol. 44, pp. 157-161, 2000. 
[4] G. L. Kung et al., "The presence of two local myocardial sheet populations confirmed by diffusion tensor MRI and histological validation," J. Magn. Reson. Imag., vol. 34, pp. 1080-1091, 2011.

[5] E. M. Tunnicliffe et al., "Intercentre reproducibility of cardiac apparent diffusion coefficient and fractional anisotropy in healthy volunteers," J. Cardiovasc. Magn. Reson., vol. 16, p. 31, 2014.

[6] P. F. Ferreira et al., "In vivo cardiovascular magnetic resonance diffusion tensor imaging shows evidence of abnormal myocardial laminar orientations and mobility in hypertrophic cardiomyopathy," J. Cardiovasc. Magn. Reson., vol. 16, p. 87, 2014.

[7] C. A. Clark and D. LeBihan, "Water diffusion compartmentation and anisotropy at high b values in the human brain," Magn. Reson. Med., vol. 44, pp. 852-859, 2000.

[8] S. D. Santis et al., "Anisotropic anomalous diffusion assessed in the human brain by scalar invariant indices," Magn. Reson. Med., vol. 65, pp. 1043-1052, 2011.

[9] S. Capuani et al., "Spatio-temporal anomalous diffusion imaging: Results in controlled phantoms and in excised human meningiomas," Magn. Reson. Imag., vol. 31, pp. 359-365, 2013.

[10] R. L. Magin, C. Ingo, L. Colon-Perez, W. Triplett, and T. H. Mareci, "Characterization of anomalous diffusion in porous biological tissues using fractional order derivatives and entropy," Micropor. Mesopor. Mat., vol. 178, pp. 39-43, 2013.

[11] M. M. Meerschaert, R. L. Magin, and A. Q. Ye, "Anisotropic fractional diffusion tensor imaging," J.Vib. Control.

[12] E. W. Hsu, D. L. Buckley, J. D. Bui, S. J. Blackband, and J. R. Forder, "Two-component diffusion tensor MRI of isolated perfused hearts," Magnet. Reson. Med., vol. 45, pp. 1039-1045, 2001.

[13] J. R. Forder, J. D. Bui, D. L. Buckley, and S. J. Blackband, "MR imaging measurement of compartmental water diffusion in perfused heart slices," Am. J. Physiol. Heart Circ. Physiol., vol. 281, pp. H1280-H1285, 2001.

[14] T. Niendorf, R. M. Dijkhuizen, D. G. Norris, M. van Lookeren Campagne, and K. Nicolay, "Biexponential diffusion attenuation in various states of brain tissue: Implications for diffusion-weighted imaging," Magn. Reson. Med., vol. 36, pp. 847-857, 1996.

[15] K. M. Bennett et al., "Characterization of continuously distributed cortical water diffusion rates with a stretched-exponential model," Magn. Reson. Med., vol. 50, pp. 727-734, 2003.

[16] D. A. Reiter et al., "Anomalous T2 relaxation in normal and degraded cartilage," Magn. Reson. Med..

[17] M. G. Hall and T. R. Barrick, "From diffusion-weighted MRI to anomalous diffusion imaging," Magn. Reson. Med., vol. 59, pp. 447-455, 2008.

[18] A. Bueno-Orovio, D. Kay, and K. Burrage, "Fourier spectral methods for fractional-in-space reaction-diffusion equations," BIT Numer. Math., vol. 54, pp. 937-954, 2014.

[19] L. Deseri and M. Zingales, "A mechanical picture of fractional-order Darcy equation," Commun. Nonlinear Sci. Numer. Simulat., vol. 20, pp. 940-949, 2015.

[20] Q. Yang, I. Turner, F. Liu, and M. Ilić, "Novel numerical methods for solving the time-space fractional diffusion equation in two dimensions," SIAM J. Sci. Comput., vol. 33, pp. 1159-1180, 2011.

[21] Q. Yu, F. Liu, I. Turner, and K. Burrage, "Numerical investigation of three types of space and time fractional Bloch-Torrey equations in 2D," Cent. Eur. J. Phys., vol. 11, pp. 646-665, 2013.

[22] W. van der Zwaag et al., "Minimization of Nyquist ghosting for echoplanar imaging at ultra-high fields based on a negative readout gradient strategy,” J. Magn. Reson. Imag., vol. 30, pp. 1171-1179, 2009.

[23] R. Garrapa, "Numerical evaluation of two and three parameters Mittag-Leffler functions," SIAM J. Numer. Anal., vol. 53, pp. 1350-1369, 2015.

[24] M. D. Cerqueira et al., "Standardized myocardial segmentation and nomenclature for tomographic imaging of the heart," Circulation, vol. 105, pp. 539-542, 2002.

[25] I. J. LeGrice et al., "Laminar structure of the heart: Ventricular myocyte arrangement and connective tissue architecture in the dog," $\mathrm{Am}$. J. Physiol., vol. 269, pp. H571-H582, 1995.

[26] S. H. Gilbert et al., "Visualization and quantification of whole rat heart laminar structure using high-spatial resolution contrast-enhanced MRI,” Am. J. Physiol. Heart Circ. Physiol., vol. 302, pp. H287-H298, 2012.
[27] O. Bernus et al., "Comparison of diffusion tensor imaging by cardiovascular magnetic resonance and gadolinium enhanced $3 \mathrm{D}$ image intensity approaches to investigation of structural anisotropy in explanted hearts," J. Cardiov. Magn. Reson., vol. 17, p. 31, 2015.

[28] A. J. Pope, G. B. Sands, B. H. Smaill, and I. J. LeGrice, "Three-dimensional transmural organization of perimysial collagen in the heart," $\mathrm{Am}$. J. Physiol. Heart Circ. Physiol., vol. 295, pp. H1243-H1252, 2008.

[29] S. H. Gilbert, A. P. Benson, P. Li, and A. V. Holden, "Regional localisation of left ventricular sheet structure: Integration with current models of cardiac fibre, sheet and band structure," Eur. J. Cardiothorac Surg., vol. 32, pp. H231-H249, 2007.

[30] S. R. M. Reynolds, M. Kirsch, and R. J. Bing, "Functional capillary beds in the beating $\mathrm{KCl}$-arrested and $\mathrm{KCl}$-arrested-perfused myocardium of the dog," Circ Res., vol. 6, pp. 600-611, 1958.

[31] J. B. Bassingthwaighte, T. Yipintsoi, and R. B. Harvey, "Microvasculature of the dog left ventricular myocardium," Microvasc. Res., vol. 7, pp. 229-249, 1974.

[32] J. Shinari, K. Berezowski, and W. C. Roberts, "Quantitative measurement of normal and excessive (cor adiposum) subepicardial adipose tissue, its clinical significance, and its effect on electrocardiographic QRS voltage," Am. J. Cardiol., vol. 76, pp. 414-418, 1995.

[33] P. A. Helm, H.-J. Tseng, L. Younes, E. R. McVeigh, and R. L. Winslow, "Ex vivo 3D diffusion tensor imaging and quantification of cardiac laminar structure," Magn. Reson. Med., vol. 54, pp. 850-859, 2005.

[34] S. Kim, G. Chi-Fishman, A. S. Barnett, and C. Pierpaoli, "Dependence on diffusion time and apparent diffusion tensor of ex vivo calf tongue and heart," Magn. Reson. Med., vol. 54, pp. 1387-1396, 2005.

[35] C. Meier, W. Dreher, and D. Leibfritz, "Diffusion in compartmental systems. I. A comparison of an analytical model with simulations," Magn. Reson. Med., vol. 50, pp. 500-509, 2003.

[36] D. S. Novikova, J. H. Jensenb, J. A. Helpernb, and E. Fieremans, "Revealing mesoscopic structural universality with diffusion," Proc. Nat. Acad. Sci. USA, vol. 111, pp. 5088-5093, 2014.

[37] C. Ingo, R. L. Magin, and T. B. Parrish, "New insights into the fractional order diffusion equation using entropy and kurtosis," Entropy, vol. 16 , pp. 5838-5852, 2014

[38] P. R. Shorten and T. K. Soboleva, "Anomalous ion diffusion within skeletal muscle transverse tubule networks," Theor. Biol. Med. Model, vol. 4, p. 18, 2007.

[39] F. Höfling and T. Franosch, "Anomalous transport in the crowded world of biological cells," Rep. Prog. Phys., vol. 76, p. 046602, 2013.

[40] F. Grinberg et al., "Non-Gaussian diffusion imaging for enhanced contrast of brain tissue affected by ischemic stroke," PLOS ONE, vol. 9, p. e89225, 2014.

[41] S. W. Anderson et al., "Characterizing non-Gaussian, high b-value diffusion in liver fibrosis: Stretched exponential and diffusional kurtosis modeling,” J. Magn. Reson. Imag., vol. 39, pp. 827-834, 2014.

[42] J. Coatléven, H. Haddar, and J. R. Li, "A macroscopic model including membrane exchange for diffusion MRI," SIAM J. Appl. Math, vol. 74, pp. 516-546, 2014.

[43] J. H. Jensen and J. A. Helpern, "MRI quantification of non-Gaussian water diffusion by kurtosis analysis," NMR Biomed., vol. 23, pp. 698-710, 2010.

[44] A. Bueno-Orovio, D. Kay, V. Grau, B. Rodriguez, and K. Burrage, "Fractional diffusion models of cardiac electrical propagation: Role of structural heterogeneity in dispersion of repolarization," J. R. Soc. Interface, vol. 11, p. 20140352, 2014.

[45] N. Cusimano, A. Bueno-Orovio, I. Turner, and K. Burrage, "On the order of the fractional laplacian in determining the spatio-temporal evolution of a space-fractional model of cardiac electrophysiology," PLoS ONE, vol. 10, p. e0143938, 2015.

[46] P. P. Mitra, P. N. Sen, and L. M. Schwartz, "Short-time behavior of the diffusion coefficient as a geometrical probe of porous media," Phys. Rev. B, vol. 47, p. 8565, 1993.

[47] V. J. Wedeen, P. Hagmann, W.-Y. I. Tseng, T. G. Reese, and R. M. Weisskoff, "Mapping complex tissue architecture with diffusion spectrum magnetic resonance imaging," Magn. Reson. Med., vol. 54, pp. $1377-1386,2005$

[48] A. Hanyga and R. L. Magin, "A new anisotropic fractional model of diffusion suitable for applications of diffusion tensor imaging in biological tissues," Proc. R. Soc. A, vol. 470, p. 20140319, 2014. 